\title{
Observation of redshifting and harmonic radiation in inverse Compton scattering
}

\begin{abstract}
Y. Sakai, ${ }^{1}$ I. Pogorelsky, ${ }^{3}$ O. Williams, ${ }^{1}$ F. O'Shea, ${ }^{1}$ S. Barber, ${ }^{1}$ I. Gadjev,${ }^{1}$ J. Duris, ${ }^{1}$ P. Musumeci, ${ }^{1}$ M. Fedurin, ${ }^{3}$ A. Korostyshevsky, ${ }^{3}$ B. Malone, ${ }^{3}$ C. Swinson, ${ }^{3}$ G. Stenby, ${ }^{3}$ K. Kusche, ${ }^{3}$ M. Babzien, ${ }^{3}$ M. Montemagno, ${ }^{3}$ P. Jacob, ${ }^{3}$ Z. Zhong, ${ }^{3}$ M. Polyanskiy, ${ }^{3}$ V. Yakimenko, ${ }^{2}$ and J. Rosenzweig ${ }^{1}$

${ }^{1}$ University of California at Los Angeles, Los Angeles, California 90095, USA

${ }^{2}$ Stanford Linear Accelerator Center, Menlo Park, California 94025, USA

${ }^{3}$ Brookhaven National Laboratory, Upton, New York 11973, USA

(Received 22 September 2013; revised manuscript received 22 February 2015; published 17 June 2015)

Inverse Compton scattering of laser photons by ultrarelativistic electron beam provides polarized $\mathrm{x}$ - to $\gamma$-ray pulses due to the Doppler blueshifting. Nonlinear electrodynamics in the relativistically intense linearly polarized laser field changes the radiation kinetics established during the Compton interaction. These are due to the induced figure- 8 motion, which introduces an overall redshift in the radiation spectrum, with the concomitant emission of higher order harmonics. To experimentally analyze the strong field physics associated with the nonlinear electron-laser interaction, clear modifications to the angular and wavelength distributions of $\mathrm{x}$ rays are observed. The relativistic photon wave field is provided by the ps $\mathrm{CO}_{2}$ laser of peak normalized vector potential of $0.5<a_{L}<0.7$, which due to the quadratic dependence of the strength of nonlinear phenomena on $a_{L}$ permits sufficient effects not observed in past 2nd harmonic study with $a_{L} \approx 0.3$ laser [M. Babzien et al., Phys. Rev. Lett. 96, 054802 (2006)]. The angular spectral characteristics are revealed using $K$-, $L$-edge, and high energy attenuation filters. The observation indicates existence of the electrons' longitudinal motion through frequency redshifting understood as the mass shift effect. Thus, the 3rd harmonic radiation has been observed containing on-axis $x$-ray component that is directly associated with the induced figure- 8 motion. These are further supported by an initial evidence of off-axis 2 nd harmonic radiation produced in a circularly polarized laser wave field. Total x-ray photon number per pulse, scattered by $65 \mathrm{MeV}$ electron beam of $0.3 \mathrm{nC}$, at the interaction point is measured to be approximately $10^{9}$.
\end{abstract}

DOI: 10.1103/PhysRevSTAB.18.060702

PACS numbers: 41.60.Ap, 03.50.De, 41.50.+h

\section{INTRODUCTION}

Laser photons of angular frequency $\omega_{L}$, when Compton or Thomson backscattered by a highly relativistic electron of Lorentz factor $\gamma_{0} \gg 1$ are Doppler blueshifted. Initial IR-optical wavelength waves thus be scattered into the $\mathrm{x}$-ray and $\gamma$-ray regime, while preserving the initial polarization of the laser field. In this optical undulator, the photon energy is boosted to

$$
4 \gamma_{0}^{2} \hbar \omega_{L},
$$

in the case of a 180 degree (head-on) collision [1], with photon scattering assumed aligned to the initial electron direction. This effect is known as relativistic Thomson (as the wavelength of the scattered light is not notably changed when the interaction is viewed in the electron's rest frame) or inverse Compton scattering (ICS). The emission at $\mathrm{x}$ to

Published by the American Physical Society under the terms of the Creative Commons Attribution 3.0 License. Further distribution of this work must maintain attribution to the author(s) and the published article's title, journal citation, and DOI. $\gamma$-ray regime has moderately narrow spectral spread inside a radiation cone within half opening angle $<1 / \gamma_{0}$, and is highly directed.

Through the production of subpicosecond $\mathrm{x}$-ray to $\gamma$-ray pulses at photon energies extending from the $\mathrm{keV}$ to $\mathrm{MeV}$ range and beyond, ICS opens up the class of laser synchrotron sources (LSS) [2] that complement conventional high-average-power synchrotron-light facilities, as well as the linac-based x-ray free-electron laser (XFEL). Pulsed ICS could be invaluable for numerous scientific uses, and its actual application for realizing a tunable, narrow-spectrum and polarized high energy photon source has been vigorously examined [2-7].

Even before discussing LSS, the ICS interaction is of high interest beyond the laboratory as a fundamental, naturally occurring process that affects understanding experimental measurements of astrophysics phenomena [8]. Scientific, medical and industrial applications of optimized ICS sources, enabled by rapid recent improvements in electron and laser beam technologies, abound. In particular, demands for compact ICS sources at hard $\mathrm{X}$-ray photon energies (10-100 keV) are increasing, with 
examples of recent applications including tomography using phase contrast imaging in biology and medicine [9], and subpicosecond resolved pump-probe exploration of solid-phase transitions through lattice diffraction [10]. Further, recently these demonstrations have accelerated the development of the high average flux systems such as bunch train configuration [11] or CW mode operation by using an energy recovery linac (ERL) [12].

Investigation on LSS is also motivated by the creation of intense polarized positron sources for $e^{-}-e^{+}$colliders [13], and by the promise owing to modern technologies of high gradient, advanced accelerator concepts, including precise sub- $\mu$ m e-beam tuning and measurements, uniquely enabled by employing the so-termed laser Thomson wire scanner [14]. Finally, at the highest energies ( $\mathrm{GeV}$ to $\mathrm{TeV})$, the concept of $e-\gamma$ and $\gamma-\gamma$ collider will provide precious experimental opportunities of direct matter production from photon field [15], thus improve the measurements noise and the energy spread and so the mass resolution.

In an intermediate photon energy scenario, which has motivated our recent studies, directed beams of $\sim 10 \mathrm{MeV} \gamma$ rays are envisioned as unique components of a mobile system for the standoff detection of nuclear materials via photofission, photodisintegration or nuclear resonance fluorescence (NRF). Especially, NRF application has implications for fundamental, fine spectral investigations of a class of nuclear levels - this newly enabled field has been described as nuclear photonics. This demand leads to the recent demonstrations of the Compton $\gamma$-photon production and its application [16,17].

Furthermore, $\mathrm{MeV}$ class inverse Compton source based on a laser wakefield accelerator to provide the $\mathrm{MeV}-\mathrm{GeV}$ electron has been also demonstrated, with the same laser pulse which drives the plasma accelerator used in the Compton collisions yielding an all-optical source of $\gamma$-ray photons [18]. This approach is similar to the context of the research presented here, which is the development of a highly efficient - through laser pulse recirculation and multiple electron beam trains-all-optical vacuum $\gamma$-ray source in which the ICS-driving electron beam with low emittance is generated by the inverse free-electron laser (IFEL) acceleration [19]. The ICS and IFEL experiments in this joint effort are both performed currently at the Brookhaven National Laboratory Accelerator Test Facility (BNL ATF).

For example, a photofission based system of interest for nuclear materials detection would demand $\gamma$ rays having maximum energy of 10-15 MeV, produced at a rate of $10^{13}$ photons/sec. Given an achievable repetition rate around $1 \mathrm{kHz}$, limited by thermal loading of optical components of intense laser and radio frequency based accelerator power systems, this implies a per-pulse yield approaching $10^{10}$ photons/shot. For use of NRF study that requires narrow band spectrum of $\Delta \omega / \omega<10^{-3}$, further modifications to extract on-axis, $\ll 1 / \gamma_{0}$, emission component is required.
The ICS photon yield per shot is estimated as, assuming approximately equal spatiotemporal dimensions of the laser and electron beam,

$$
N_{\mathrm{ICS}} \approx c \sigma_{\mathrm{Th}} n_{L} N_{e} \Delta t_{\mathrm{ICS}}[\text { photons/shot] }
$$

This yield is proportional to the Thomson scattering cross section, $\sigma_{\mathrm{Th}}=0.67 \times 10^{-24} \mathrm{~cm}^{2}$, the number density of laser photons $n_{L}$, the number of electrons per bunch $N_{e}$, and the ICS interaction time, $\Delta t_{\mathrm{ICS}}$, which is of the order of Rayleigh range $Z_{\mathrm{Ra}} \approx \pi w_{0}^{2} / \lambda_{L}$ divided by $c$,

$$
\Delta t_{\mathrm{ICS}} \approx Z_{\mathrm{Ra}} / c .
$$

Assuming typical electron beam parameters of the BNL ATF, and the existing picosecond pulse length ATF $\mathrm{CO}_{2}$ laser of several Joule per shot: $N_{e} \approx 6 \times 10^{9}$, a $\sim$ nC electron bunch; $\Delta t_{\mathrm{ICS}} \approx$ a few picoseconds; $n_{L} \approx 10^{26}$ photons $/ \mathrm{cm}^{3}$ obtained with laser waist of $w_{0} \approx 10 \mathrm{~s} \mu \mathrm{m}$, by using a $f / 1$ optic of $2 w_{0} \approx(f / \#)\left(4 \lambda_{L} / \pi\right)$, and Eq. (3) gives a corresponding ICS $\mathrm{x}$ ray yields near $10^{10}$ photons/shot (one photon per one electron).

The peak energy density of the laser field is approximated as

$$
n_{L} \approx\left(E_{L}^{2} / 4 \pi\right) / \hbar \omega_{L},
$$

where $E_{L}$ is the laser field's amplitude. This photon density corresponds to a $\mathrm{CO}_{2}$ laser field, $E_{L} \approx 1 \mathrm{GV} / \mathrm{cm}$, which given the long wavelength $(10.6 \mu \mathrm{m})$ of the laser, produces a significant oscillatory motion in the electrons. A useful measure of this amplitude is the normalized vector potential, $a_{L}=e E_{L} / m_{e} c \omega_{L}$, which approaches unity in BNL's case. The condition $a_{L} \approx 1$ indicates that the laser is intense enough to drive transverse motion corresponding to a momentum $m_{e} c$; the oscillating and therefore radiating electrons enter into a regime of relativistic transverse motion thus giving a radiation redshift. Here the transverse oscillations become nonsinusoidal, and the existence of non-negligible effects due to the magnetic fields of the laser induce the nonlinear figure- 8 motion illustrated in Fig. 1 $[20,21]$ and described in the following section.

Qualitatively, this motion induces three notable effects. First, the existence of relativistic transverse momentum indicates that the electron must on average slow its propagation in $z$, thus giving a redshift that could be significant,

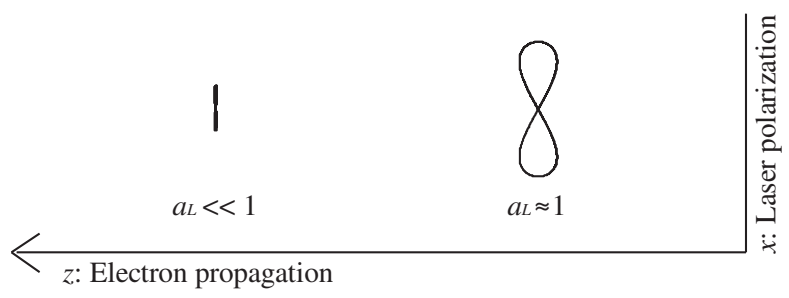

FIG. 1. Nonlinear figure-8 motion during Compton interaction. 
given the sensitivity of the relativistic Doppler boost. Second, the increasingly nonsinusoidal transverse motion in the nonlinear limit $\left(a_{L} \approx 1\right.$ or greater) indicates that strong harmonic components should be present in the radiated spectrum. Finally, the directionality of the motion-the presence of the longitudinal components that is not negligible in respect to the average longitudinal momentum in the oscillation-gives rise to unique signatures in the radiation angular spectrum, particularly in the harmonics. In this paper, experimental results describing these effects are reported.

In order to appreciate the leap forward in understanding, in terms of both the quantum mechanical [20,21] and classical [22] descriptions of the electrodynamics obtained in the measurement, we should discuss briefly the previous progress down the path. Indeed, there have been, during recent decades with actual availability of the ultrashort intense lasers, numerous detailed theoretical investigations of the characteristics of laser-induced electron motion and corresponding radiation effects [23-27]. However, only few experimental activities were carried out in this field [28-31]. The first foray into the nonlinear regime of ICS reported in Refs. [32,33] displayed necessarily production of the 2nd harmonic. This paper thus would represent a sufficient confirmation of the essential features of the nonlinear interaction.

These physics issues concern the fundamental electrodynamics aspects. They also have a practical side, as the details of the spectrum produced greatly affect the prospects for applications. At the most basic level, one must understand the implications of redshifting and attendant spectral broadening, as well as the generation of higher order harmonics due to nonlinear effects when considering applications such as nuclear fluorescence-based detection schemes. At a more detailed level, these nonlinear issues should be understood to aid in producing and optimizing the modulations of the temporal and spectral characteristics of the scattered $\mathrm{x}$ rays produced. This understanding may lead to development of more sophisticated variants of the nonlinear ICS, including schemes based on a two-laserfrequency interaction (or mixing) at $x$ to $\gamma$-ray regime [34].

\section{CLASSICAL DESCRIPTION ON RADIATION KINETICS OF LINEARLY POLARIZED ICS}

In order to provide the proper intuition to appreciate the salient observations, it is necessary to review and simplify the classical theory of the inverse Compton scattering radiation process in the experimental aspect.

In remainder of this paper, the above-mentioned laser amplitude parameter, $a_{L}$, is redefined as the peak vector potential of the laser, normalized to the electron's rest mass [21],

$$
a_{L} \equiv a_{L, 0, \text { peak }} \equiv \frac{\sqrt{-e^{2} A_{\mu} A^{\mu}}}{m_{e} c^{2}}=\frac{e E_{L, 0}\left(\lambda_{L} / 2 \pi\right)}{m_{e} c^{2}},
$$

where $A_{\mu}$ is the peak vector potential, $E_{L, 0}$ is the peak electric field amplitude of a laser that is assumed linearly polarized in the $x$ direction, and $\lambda_{L}$ is the laser wavelength. This definition describes that, when $a_{L}$ approaches unity, the transverse motion of the electron takes on relativistic character, since an electron obtains the equivalent energy of its rest mass in one optical cycle. Further, it is clear that if one wishes to experimentally induce strong transverse motion, in the sense of large $a_{L}$, it is advantageous to use a long wavelength $\lambda_{L}$ laser to obtain a larger acceleration path. This corresponds to increase of the photon number density $n_{L}$ and thus the scattered flux, at the expense of a proportionately lower ICS photon energy.

The most intuitive effects of a strong laser field on the emitted ICS radiation is that, as noted above, the fundamental spectrum acquires a frequency redshift [21],

$$
\omega_{\mathrm{ICS}, 1 \mathrm{st}} \approx \frac{4 \gamma_{0}^{2} \omega_{L}}{1+\frac{a_{L}^{2}}{2}+\gamma_{0}^{2} \Theta^{2}},
$$

where pursuant to the previous use $\gamma_{0}$ is defined by ultrarelativistic electron beam energy by $\mathcal{E}_{0}=\gamma_{0} m_{e} c^{2}$. Here the limit that $\Theta<1 / \gamma$ is the observation angle relative to the initial electron beam propagation axis is taken. The on-axis redshift, with $\Theta=0$, is understood as the decrease in the longitudinal electron velocity due to its redirection into transverse oscillation, or alternatively as an effective mass increase; the electron is "dressed" by the vector potential, and obtains oscillatory transverse momentum $p_{L, x}=e A_{x} / c$. This point of view is expressed as an effective mass increase as [21]

$$
m_{e, \mathrm{eff}}^{2} \equiv m_{e}^{2}+\left(\overline{\frac{p_{L, x}}{c}}\right)^{2}=m_{e}^{2}\left\{1+\left(\frac{a_{L}}{\sqrt{2}}\right)^{2}\right\}
$$

resulting in reduction of effective Lorenz factor,

$$
\gamma_{\mathrm{eff}} \equiv \frac{m_{e}}{m_{e, \mathrm{eff}}} \gamma_{0}
$$

In the nonlinear limit, viewed classically, the nonsinusoidal deflection of the electron trajectory by the action of the laser field approaches or exceeds $1 / \gamma_{0}$, and the radiation kinetics becomes complex. This results in notable changes in the angular spectrum of the radiation. The nonsinusoidal electron motion due to the $v_{x} \times B$ acceleration is induced, and thus a phase delay between electron motion and the purely sinusoidal colliding laser wave as a photon source is initiated. This nonlinear electrodynamics scenario in turn induces, as noted above, classical production of harmonic radiation; in quantum mechanical terms, these processes are viewed as due to multiphoton absorption in dense photon field, and subsequent reemission of one photon of higher photon energy. Each harmonic has a characteristic 
angular radiation pattern keeping the axial symmetry in oscillating laser wave field.

In the case of a linearly polarized laser field, the electron oscillation displays the figure 8 shape (Fig. 1) as viewed in the electron beam's nominal rest frame. Indeed, the components of the motion in the longitudinal (along the beam propagation) direction are represented in the Galilean frame moving with the electron beam, in the small angle approximation as

$$
\left.\begin{array}{l}
v_{z}=v_{0} \cos \theta \cong v_{0}\left\{1-\frac{1}{2}\left(\frac{e A_{x}}{\mathcal{E}_{0}}\right)^{2}\right\}, \\
v_{x}=v_{0} \sin \theta \cong v_{0} \frac{e A_{x}}{\mathcal{E}_{0}},
\end{array}\right\}
$$

where $\theta=\theta(t)$ is the propagation angle of the oscillating electron written as

$$
\sin \theta \cong \frac{p_{L, x}}{p_{z}} \ll 1, \quad p_{z} \cong \frac{\mathcal{E}_{0}}{c},
$$

with $v_{0}$ an initial ultrarelativistic electron's velocity, as expressed to the second order in $\gamma_{0}{ }^{-1}$,

$$
v_{0} \cong c\left(1-\frac{1}{2 \gamma_{0}^{2}}\right) .
$$

In the nominal Galilean frame of the electron, the laser vector potential is $A_{x}=A \cos \left(\omega_{e, L} t\right)$, having angular frequency $\omega_{e, L} \cong 2 \omega_{L}$ by counter collision. Here, $v_{z}$ is the longitudinal velocity and $v_{x}$ is the transverse velocity in the lab flame. In this analysis, small angle approximation is utilized as our experimental interest is in the vicinity of the relativistic threshold with $a_{L} \leq 1$.

The radiation dynamics occurring during the ICS laserelectron interaction may be understood by viewing the electron as a radiator stimulated most effectively by the incident photon when its motion is directed toward the observer, through a relativistic boost. This conclusion is reached by examining the classical far-field LienardWiechert (LW) potential, as it is seen along the observation direction $\mathbf{n}$. For $\omega_{\text {ICS }} \ll c / r_{e}$, the LW potential yields the field [35]

$$
e \boldsymbol{E}_{\mathrm{LW}}=m_{e} \frac{r_{e}}{R} \frac{\mathbf{n} \times\left\{\left(\mathbf{n}-\frac{v}{c}\right) \times \boldsymbol{w}\right\}}{\left(1-\mathbf{n} \cdot \frac{v}{c}\right)^{3}}
$$

Here $\boldsymbol{w}$ is the acceleration vector. For small angles between the velocity and observation vector, $\Delta \Theta$, and under the assumption of the ultrarelativistic condition, $\Delta \Theta<1 / \gamma_{0} \ll 1$, Eq. (12) is approximately reduced to the following field, with, $\mathbf{n} \rightarrow(\Delta \Theta, 0,1)$, which is proportional to the polarization vector $\hat{\mathbf{x}}$,

$$
\boldsymbol{E}_{\mathbf{L W}} \propto-\frac{w_{x}\left(1-\frac{v_{z}}{c}\right)-w_{z}\left(\Delta \Theta-\frac{v_{x}}{c}\right)}{\left(1-\Delta \Theta \frac{v_{x}}{c}-\frac{v_{z}}{c}\right)^{3}} \hat{\mathbf{x}} .
$$

The retarded field of Eq. (13) has strong maxima when $\Delta \Theta \rightarrow 0$ and the longitudinal velocity, $v_{z}(t)$, becomes comparable to the speed of light during the relativistic figure-8 motion.

In Eq. (13), $v_{z}=$ constant with $w_{z}=0$, corresponds to the linear Thomson scattering of a head-on energetic electron-photon collision. In this case $\boldsymbol{E}_{\mathrm{LW}}$ becomes a function only of $w_{x}$ for the observation angle $\Delta \Theta=0$. On the other hand, for the nonlinear case, with $v_{z}(t) \neq$ constant, the effect of both longitudinal and transverse acceleration components induced by the figure- 8 motion should be examined. In this way, it can be seen that near-axis harmonic radiation $(\Delta \Theta=0)$ associated with figure- 8 motion is induced by the oscillatory behavior in $v_{z}$. This component produces, due to the symmetry of the field oscillation, only odd harmonic radiation. On the other hand, off-axis harmonic radiation with $\Delta \Theta \neq 0$ is function of both $v_{x}(t)$ and $v_{z}(t)$, thus permits other symmetries in the kinetics, to induce both odd and even harmonics. These results have been noted in the related phenomena in high strength magnetostatic undulators in FELs, general synchrotron light sources.

\section{A. Remarks on ICS x-ray angular spectrum}

Fourier transformation of the Lienard-Wiechert field yields the frequency spectrum by integrating the acceleration terms in Eq. (12), and extracting the number of emitted photon modes per unit $d \omega, \mathrm{d} N_{\omega}=4 \pi p^{2} \mathrm{~d} p / h^{3}=$ $\omega^{2} \mathrm{~d} \omega / 2 c^{3} \pi^{2}$, as [36,37],

$\frac{d I}{d \boldsymbol{\Omega}}=d N_{\omega} c \pi\left|\frac{1}{\sqrt{2 \pi}} \int_{-\infty}^{\infty} \mathbf{n} \times(\mathbf{n} \times \boldsymbol{v}) e^{i \omega\left(t-\frac{\mathrm{n} \cdot r}{c}\right)} d t\right|^{2} \alpha \hbar$

where $\alpha$ is the fine structure constant. Using the Eq. (9) of figure-8 motion an electron's position is integrated within the colliding laser cycle to give

$$
\begin{array}{r}
z=\int v_{z} d t \cong v_{0}\left\{1-\frac{1}{4}\left(\frac{a_{L}}{\gamma_{0}}\right)^{2}\right\} t-\frac{v_{0}}{4}\left(\frac{a_{L}}{\gamma_{0}}\right)^{2} \frac{\sin 2 \omega_{e, L} t}{2 \omega_{e, L}} \\
\cong c\left(1-\frac{1+a_{L}^{2} / 2}{2 \gamma_{0}^{2}}\right) t-\frac{c}{4}\left(\frac{a_{L}}{\gamma_{0}}\right)^{2} \frac{\sin 2 \omega_{e, L} t}{2 \omega_{e, L}}, \\
x=\int v_{x} d t \cong v_{0} \frac{a_{L}}{\gamma_{0}} \frac{\sin \omega_{e, L} t}{\omega_{e, L}} \cong c \frac{a_{L}}{\gamma_{0}} \frac{\sin \omega_{e, L} t}{\omega_{e, L}} .
\end{array}
$$

Here the peak normalized vector potential is approximated as $a_{L}=e A / m_{e} c^{2}$ and high order terms of $a_{L} / \gamma_{0}$ are neglected. The retarded term $\exp \omega(1-\mathbf{n} \cdot \boldsymbol{r} / c)$ in the LW spectrum of Eq. (14) is rewritten by 


$$
\begin{aligned}
e^{i \omega\left(t-\frac{\mathbf{n} \cdot r}{c}\right)} & =e^{i \omega\left(t-\frac{z}{c}-\Delta \Theta \frac{r}{c}\right)} \\
& =e^{i \omega\left(\frac{1+a_{L}^{2} / 2}{2 \gamma_{0}^{2}}\right)} e^{i \omega\left(\frac{1}{4}\left(\frac{a_{L}}{\gamma_{0}}\right)^{2} \frac{\sin 2 \omega_{e, L} t}{2 \omega_{e, L}}\right)} e^{i \omega\left(\Delta \Theta_{\frac{a_{L}}{\gamma_{0}} \sin \omega_{e, L} \omega_{e, L}}\right)}
\end{aligned}
$$

In addition, the expression $\mathbf{n} \times(\mathbf{n} \times \boldsymbol{v})$ in Eq. (14) is reduced to $\left(-v_{x}+\Delta \Theta v_{z}\right) \hat{\mathbf{x}}-\Delta \Theta\left(-v_{x}+\Delta \Theta v_{z}\right) \hat{\mathbf{z}} \cong$ $-v_{x} \hat{\mathbf{x}} \cong c \frac{a_{L}}{\gamma_{0}} \operatorname{Re}\left(e^{i \omega_{e, L} t}\right) \hat{\mathbf{x}}$, with the ultrarelativistic condition $\Delta \Theta<1 / \gamma_{0} \ll 1$ assumed. Further, by an expansion utilizing a Bessel function of the first kind, $e^{i b \sin \sigma}=\sum \boldsymbol{J}_{n}(b) e^{i n \sigma}$, $\boldsymbol{J}_{n}(b)=\sum_{k=0}^{\infty} \frac{(-1)^{k} b^{2 k+n}}{2^{2 k+n} k !(k+n) !}$, the double differential spectrum for the nonlinear ICS radiation is resolved as

$$
\begin{aligned}
\frac{d^{2} I}{d \omega d \Omega} \approx & \frac{e^{2} \omega^{2}}{4 \pi^{2} c}\left(\frac{a_{L}}{\gamma_{0}}\right)^{2} \\
\times & \times \begin{array}{c}
\times \sum_{n_{z}=-\infty}^{\infty} J_{z, n_{z}}\left[\frac{1}{4}\left(\frac{a_{L}}{\gamma_{0}}\right)^{2} \frac{\omega}{2 \omega_{e, L}}\right] e^{i n_{z} 2 \omega_{e, L} t} \\
\times \sum_{n_{x}=-\infty}^{\infty} J_{x, n_{x}}\left(\Delta \Theta \frac{a_{L}}{\gamma_{0}} \frac{\omega}{\omega_{e, L}}\right) e^{i n_{x} \omega_{e, L} t}
\end{array} \mid
\end{aligned}
$$

Here the indices $n_{x}$ and $n_{z}$ are associated with the harmonics of the transverse and longitudinal motion respectively. The angular frequency of ICS photon $\omega$ is evaluated through the condition

$$
e^{i\left(1+2 n_{z}+n_{x}\right) \omega_{L} t} e^{i\left(\frac{1+a_{L}^{2} / 2}{4 \gamma_{0}^{2}}\right) \omega t}=1 .
$$

Here angular frequency is reverted back to examine the experimental observable, the laser's angular frequency in the lab frame, that is $\omega_{e, L}=2 \omega_{L}$. For small values of their arguments, the first three Bessel functions of first kind are approximated by

$$
\left.\begin{array}{l}
\boldsymbol{J}_{0}(b)=\sum_{k=0}^{\infty}(-1)^{k} \frac{\left(b^{2} / 4\right)^{k}}{(k !)^{2}} \approx 1-\frac{b^{2}}{4}<1, \\
\boldsymbol{J}_{1}(b)=\sum_{k=0}^{\infty}(-1)^{k} \frac{b^{2 k+1}}{2^{2 k+1} k !(k+1) !} \approx \frac{b}{2}-\frac{b^{3}}{16}<0.6, \\
\boldsymbol{J}_{2}(b)=\sum_{k=0}^{\infty}(-1)^{k} \frac{b^{2 k+2}}{2^{2 k+2} k !(k+2) !} \approx \frac{b^{2}}{8}-\frac{b^{4}}{96}<0.5 .
\end{array}\right\}
$$

\section{Fundamental harmonic radiation}

The radiation pattern of the first harmonic is given by Bessel function of the first kind at order $n_{x}=n_{z}=0$, with the resulting double differential spectrum observed:

$$
\frac{d^{2} I_{1 \mathrm{st}}}{d \omega d \Omega} \propto \omega^{2}\left|\begin{array}{c}
e^{i \omega_{e, L} t} e^{i\left(\frac{1+a_{L}^{2} / 2}{2 \gamma_{0}}\right) \omega t} \\
\times \boldsymbol{J}_{z, 0}\left[\frac{1}{4}\left(\frac{a_{L}}{\gamma_{0}}\right)^{2} \frac{\omega}{2 \omega_{e, L}}\right] \boldsymbol{J}_{x, 0}\left(\Delta \Theta \frac{a_{L}}{\gamma_{0}} \frac{\omega}{\omega_{e, L}}\right)
\end{array}\right|^{2} .
$$

In Eq. (21) the factor $\boldsymbol{J}_{z, 0} \boldsymbol{J}_{x, 0}$ may be further approximated as, neglecting higher orders of $a_{L} / \gamma_{0}$ in the case of low $a_{L}$,

$$
\left|\boldsymbol{J}_{z, 0}\left(b_{z}\right) \boldsymbol{J}_{x, 0}\left(b_{x}\right)\right|^{2} \approx 1-\frac{1}{2} b_{z}^{2}-\frac{1}{2} b_{x}^{2} .
$$

Thus Eq. (21) has on-axis maxima at $\Delta \Theta=0$ when $\boldsymbol{J}_{x, 0}(0)=1$. An increase of $a_{L}$ results in decreases of both the $\boldsymbol{J}_{z, 0}$ and $\boldsymbol{J}_{x, 0}$ components, so that radiation energy is converted to the higher order harmonics. The frequency redshift due to the electron's longitudinal position is indicated in the exponential term explicitly, $\exp \left[i\left(\frac{1+a_{L}^{2} / 2}{2 \gamma_{0}{ }^{2}}\right) \omega t\right]$, as anticipated in the undulator equation expression in Eq. (6).

Note that the off-axis intensity drop in the fundamental harmonic component is partially due, noting the off-axis redshift effect, to decrease of photonic mode density $d N_{\omega} \propto \omega^{2}$. As seen by the noted behavior of $\boldsymbol{J}_{x, 0}$, the intensity drop along the radiation angle $\Delta \Theta$ along the laser's polarization direction is more notable for a higher $a_{L}$, and thus the radiation pattern becomes elliptical, having a narrower opening angle in laser polarization direction. This effect results in conversion of off-axis radiation energy into the higher order harmonics due to nonlinear motion described by the Bessel terms. Note that for the case of $a_{L}>1$, where this small angle approximation is not valid, radiation distribution shows two lobes described by antenna type dipole Lorentz boost.

\section{Second harmonic radiation}

The 2nd harmonic radiation spectral distribution is represented by $n_{z}=0, n_{x}=1$ component in Eq. (18),

$\frac{d^{2} I_{2 \mathrm{nd}}}{d \omega d \Omega} \propto \omega^{2}\left|\begin{array}{c}e^{i 2 \omega_{e, L} t} e^{i\left(\frac{1+a_{L}{ }^{2} / 2}{2 \gamma_{0}{ }^{2}}\right) \omega t} \\ \times \boldsymbol{J}_{z, 0}\left[\frac{1}{4}\left(\frac{a_{L}}{\gamma_{0}}\right)^{2} \frac{\omega}{2 \omega_{e, L}}\right] \boldsymbol{J}_{x, 1}\left(\Delta \Theta \frac{a_{L}}{\gamma_{0}} \frac{\omega}{\omega_{e, L}}\right)\end{array}\right|^{2}$.

Since $\boldsymbol{J}_{x, 1}$ has a maximum value $\boldsymbol{J}_{1}(2) \approx 0.6$, the angle of peak intensity for $a_{L} \approx 1$ is in the vicinity of

$$
\Delta \Theta \frac{a_{L}}{\gamma_{0}} \frac{2 \times 2 \omega_{e, L} \gamma_{0}^{2}}{\omega_{e, L}} \approx 2 \leftrightarrow \Delta \Theta \approx \frac{1}{2 \gamma_{0}} .
$$

The radiation pattern in the 2 nd harmonic is formed by two crescent shaped lobes. The spectral intensity is again proportional to the density of photonic modes, $d N_{\omega} \propto \omega^{2}$, neglecting higher order terms of $a_{L} / \gamma_{0}$. This results in decrease of the opening angle less than the value $1 /\left(2 \gamma_{0}\right)$ of Eq. (24).

The relative intensity of 2 nd harmonic with respect to fundamental grows at off-axis observation angles. The $\boldsymbol{J}_{z, 0} \boldsymbol{J}_{x, 0}$ term in Eq. (22) in this case reduces to 


$$
\left|\boldsymbol{J}_{z, 0}\left(b_{z}\right) \boldsymbol{J}_{x, 0}\left(b_{x}\right)\right|^{2} \approx 1-\frac{1}{2} b_{x}^{2} \rightarrow 1-\frac{1}{2} a_{L}^{2} .
$$

This is straightforwardly obtained by taking $\Delta \Theta \rightarrow$ $1 /\left(2 \gamma_{0}\right)$ and $\omega \approx 1 \times 2 \gamma_{0}^{2} \omega_{e, L}$. While, under these conditions, the 2nd harmonic intensity is approximately proportional to

$$
\left|\boldsymbol{J}_{z, 0}\left(b_{z}\right) \boldsymbol{J}_{x, 1}\left(b_{x}\right)\right|^{2} \approx \frac{b_{x}^{2}}{4} \rightarrow \frac{1}{4} a_{L}^{2} .
$$

The reduction of $\boldsymbol{J}_{x, 0}$ is largely opposed by the behavior of the $\boldsymbol{J}_{x, 1}$ term. As long as $a_{L}<1$, in which case the 2 nd harmonic intensity dominates that of the higher order harmonics, the intensity lost in fundamental radiation band is largely due to the radiation energy emitted in the 2nd harmonic. To guide intuition in the understanding the present experimental implementation herein, the value of the Bessel function product is roughly evaluated to be 0.9 for $a_{L} \approx 0.6$ case, and approximately $\sim 10 \%$ of total ICS radiation intensity is expected to be appear in the 2 nd harmonic component. For higher values of $a_{L}$, this approach to estimation should not be valid due to significant increase of higher order harmonics.

\section{Third harmonic radiation}

The on-axis component of the 3rd harmonic noted is attributed to the longitudinal velocity modulation term $v_{z}(t)$ given by

$$
\frac{d^{2} I_{3 \mathrm{rd}, \text { on-axis }}}{d \omega d \Omega} \propto \omega^{2} \mid \times \boldsymbol{J}_{z, 1}\left[\frac { 1 } { 4 } \left(\left.\frac{\left.e^{i 3 \omega_{e, L} t} e^{\left.i\left(\frac{a_{L}}{\gamma_{0}}\right)^{2} \frac{\omega}{2 \omega_{e, L}}\right]}\right] \boldsymbol{J}_{x, 0}\left(\Delta \Theta \frac{a_{L}{ }^{2} / 2}{\gamma_{0}} \frac{\omega}{\omega_{e, L}}\right)}{\mid 2}\right|^{2}\right.\right.
$$

The radiation's opening angle is estimated by expanding the Bessel function, $\boldsymbol{J}_{x, 0}$ and setting the frequency appropriately to $\omega \approx 3 \times 2 \gamma_{0}^{2} \omega_{e, L}$, to yield

$\boldsymbol{J}_{x, 0}\left(\Delta \Theta \frac{a_{L}}{\gamma_{0}} \frac{\omega}{\omega_{e, L}}\right) \approx 1-\frac{1}{4} b_{x}^{2} \approx 1-\left(\Delta \Theta 3 \gamma_{0} a_{L}\right)^{2}$.

This term vanishes for the angle $\Delta \Theta \approx 1 / 3 \gamma_{0} a_{L}$. For $a_{L} \approx 1$, radiation is confined within

$$
\Delta \Theta \approx 1 /\left(3 \gamma_{0}\right) .
$$

The mode density factor $d N_{\omega}$ decreases this angle further. This aspect is generally advantageous in a directed light source based on an odd order higher harmonic. The ratio of on-axis, $\Delta \Theta=0$, intensity of the $3 \mathrm{rd}$ harmonic to the peak off-axis intensity of 2 nd harmonic component is roughly approximated as

$$
\begin{aligned}
\left|\frac{\frac{d^{2} I_{3 \mathrm{rd}}}{d \omega \omega \Omega}}{\frac{d^{2} I_{2 \mathrm{nd}}}{d \omega d \Omega}}\right| & \approx\left(\frac{3 \omega_{e, L}}{2 \omega_{e, L}}\right)^{2}\left|\frac{\boldsymbol{J}_{z, 1}\left(b_{z, 3 \mathrm{rd}}\right) \times 1}{\boldsymbol{J}_{z, 0}\left(b_{z, 2 \mathrm{nd}}\right) \times 0.6}\right|^{2} \\
& \approx \frac{9}{4}\left|\frac{1}{0.6} \frac{\frac{b_{z, 3 \mathrm{rdd}}}{2}-\frac{b_{z, 3 \mathrm{rd}}{ }^{3}}{16}}{1-\frac{b_{z, \text { ndd }}{ }^{2}}{4}}\right|^{2} \approx \frac{9}{4}\left|\frac{1}{0.6} \frac{\frac{b_{z, 3 \mathrm{rd}}}{2}}{1}\right|^{2} \rightarrow a_{L}{ }^{4},
\end{aligned}
$$

for $a_{L} \leq 1$, where the limiting relation,

$$
b_{z, 3 \mathrm{rd}} \rightarrow \frac{3}{4} a_{L}^{2},
$$

is utilized to yield intuitive approximations relevant to the experiment. This ratio reaches $\sim 10 \%$ for the $a_{L} \approx 0.6$ case, and is experimentally observable, as is clarified by the experimental data. In the experiment reported in Refs. [32,33] that the ratio is $\sim 2.5 \%$, and the 3rd harmonic, with absolute value of the radiation flux $\sim 64$ times smaller, could not be examined.

The off-axis components of the 3rd harmonic are composed of two lobes and is expressed by

$\frac{d^{2} I_{3 \mathrm{rd}, \text { off-axis }}}{d \omega d \Omega} \propto \omega^{2}\left|\times \boldsymbol{J}_{z, 0}\left[\frac{1}{4}\left(\frac{e_{L}}{\gamma_{0}}\right)^{2} \frac{\omega}{2 \omega_{e, L} t} e^{i\left(\frac{1+a_{L}{ }^{2} / 2}{2 \gamma_{0}{ }^{2}}\right) \omega t}\right] \boldsymbol{J}_{x, 2}\left(\Delta \Theta \frac{a_{L}}{\gamma_{0}} \frac{\omega}{\omega_{e, L}}\right)\right|^{2}$.

Here, $\boldsymbol{J}_{x, 2}\left(b_{x}\right)$ has maximum value $\boldsymbol{J}_{2}(3) \approx 0.5$, to give a radiation opening angle of

$$
\Delta \Theta \frac{a_{L}}{\gamma_{0}} \frac{3 \times 2 \omega_{e, L} \gamma_{0}^{2}}{\omega_{e, L}} \approx 3 \leftrightarrow \Delta \Theta \approx \frac{1}{2 \gamma_{0}} .
$$

\section{B. Numerical illustrations based on $E_{\mathrm{LW}}$ of Eq. (12)}

Figure 2 shows the angular patterns of the photon number density distributions, $\left|\boldsymbol{E}_{\mathbf{L W}}\right|^{2} / \hbar \omega$, of the ICS $\mathrm{x}$ rays for the fundamental, 2nd and 3rd harmonic energy bands, respectively, for interaction parameters relevant to the experiment. The numerical calculations are based on the LW fields of Eq. (12),

$$
\begin{aligned}
\left|\boldsymbol{E}_{\mathrm{LW}}(\omega)\right|^{2}= & \left|\int_{-\infty}^{\infty} E_{\mathrm{LW}, z}(\omega) e^{i \omega t} d t\right|^{2} \\
& +\left|\int_{-\infty}^{\infty} E_{\mathrm{LW}, x}(\omega) e^{i \omega t} d t\right|^{2},
\end{aligned}
$$

under the momentum-energy 4-vector amplitude conservation and the Lorentz force equation,

$$
\left.\begin{array}{c}
p_{\mu} p^{\mu}=m_{e} c^{2} \\
\frac{d p}{d t}=e \boldsymbol{E}_{L}+e\left(\frac{v}{c}\right) \times \boldsymbol{H}
\end{array}\right\}
$$




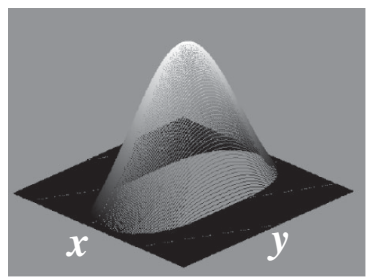

(a)

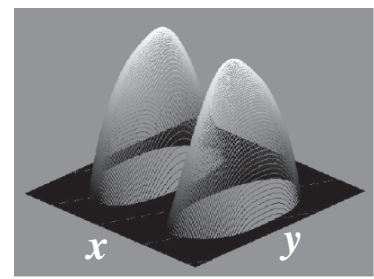

(b)

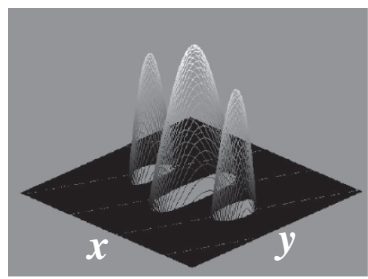

(c)

FIG. 2. Numerically calculated normalized photon number density distributions having $x$ and $z$ linearly polarized components, $\sim\left|\boldsymbol{E}_{\mathbf{L W}}\right|^{2} / \hbar \omega$, for 1 st $0<\hbar \omega<7.6 \mathrm{keV}$ (a), 2nd $7.6<\hbar \omega<15.2 \mathrm{keV}$ (b), and 3rd $15.2<\hbar \omega<22.8 \mathrm{keV}$ (c) harmonics in $16 \times 16 \mathrm{mrad}$ square where $a_{L}=0.6$ and $\mathcal{E}_{0}=65 \mathrm{MeV}$. The direction of the laser's polarization is along the $x$ axis.

The electron's nonlinear motion is solved for an ideal sinusoidal wave field, $E_{L}=E_{L, 0} \sin (k z-\omega t)$ and the observation plane is taken to be at unit length $R(t) \approx 1 \mathrm{~m}$, which is well past the radiation formation length and thus into the far field. For relevance to the subsequent discussions, the parameters used for the laser and electron beams in this numerical calculation are for this experimental condition of laser normalized vector potential of $a_{L}=0.6$ and e-beam initial energy of $\mathcal{E}_{0}=65 \mathrm{MeV}$.

\section{EXPERIMENTAL SETUP}

An initial observation of nonlinear effects in the ICS interaction, in the form of evidence for 2 nd harmonic $x$ rays, was achieved in single-shot measurements at the BNL ATF using an infrared $\mathrm{CO}_{2}$ laser [32,33] with estimated normalized vector potential of $a_{L} \approx 0.3$. In this paper, with the availability of 4 times the laser intensity, the laser vector potential has been doubled to approximately $a_{L} \approx 0.6$ [38]. The concomitant quadrupling of the strength the coupling to relevant nonlinear phenomena has permitted us to approach the relativistic threshold $a_{L}=1-$ redshifting and higher harmonic production. This fourfold increase in nonlinear interaction strength permits the robust characterization of the angular distribution of the ICS x ray. In this experiment, a $\sigma \approx 3 \mathrm{ps}, 0.3 \mathrm{nC}$ total charge, $\mathcal{E}_{0}=$ $65 \mathrm{MeV}$ electron beam derived from a rf photoinjector and linac is collided with a $\sim 5 \mathrm{ps}$ FWHM [38] $\mathrm{CO}_{2}$ laser pulse having $2-4 \mathrm{~J}$ average energy. This laser thus has approximately $\sim 0.4$ TW peak power, and with $2 w_{0} \approx 100 \mu \mathrm{m}$, thus normalized vector potentials of $a_{L} \approx 0.6$ is expected. As illustrated in Fig. 3, the $\mathrm{CO}_{2}$ laser optics inside the vacuum electron-beam line consist of two $\mathrm{Cu}$ mirrors, a $45^{\circ}$ deflector, and an off-axis parabola used for $f / 1$ laser focusing. Off-axis parabola has $1.6 \mathrm{~mm}$-diameter $(25 \mathrm{mrad}>2 / \gamma)$ axial holes for transmitting the electron and ICS x-ray beams. The spot size of the incident $\mathrm{CO}_{2}$ laser at the $45^{\circ}$ deflecting mirror is $\sim 40 \mathrm{~mm}$, and the measured beam waist at the IP is indeed $w_{0}=40 \mu \mathrm{m}$ with $Z_{\mathrm{Ra}}=0.5 \mathrm{~mm}$ at the oscillator signal level. To detect the far-field angular pattern of radiation, an x-rayoptimized potassium bromide-coated microchannel plate system (PHOTONIS MCP 40/12/10/8 I 60:1 EDR KBR 6" FM P46) with a $\sim 25 \mathrm{~mm}$ diameter (the minimum diameter of the system is restricted by the presence of vacuum pipe boundaries and related concerns), in combination with a phosphor screen and a 12-bit CCD camera (Basler, scA1400-17 gm), is employed. The distance from the IP to the MCP is $1.85 \mathrm{~m}$, giving a collection angle of $13.5 \mathrm{mrad}(>1 / \gamma \approx 8 \mathrm{mrad})$. The total X-ray flux is measured by use of a $\mathrm{Si}$ diode detector (Canberra, A300, with use of 0.5, 2.2 or $10 \mathrm{nF}$ capacitors.) that had been calibrated at the photon energies of 6,12 , and $18 \mathrm{keV}$, respectively, on BNL NSLS X15A beam line.

The features of the radiation characteristics have been resolved through the use of a MCP in tandem with judiciously chosen $\mathrm{x}$-ray filters. These filters exploit $K$ and $L$ - edge attenuation in metallic foils [39] to obtain wavelength-angular spectral information following the method studied in Ref. [40]. These foils act as low-pass or band-pass filters (as illustrated in Fig. 4), depending on the material and thickness selected. For narrow band spectral discrimination, thin foils composed of $\mathrm{Fe}$ and $\mathrm{Au}$ were utilized, having absorption edges $(K$ - and $L$-) at energies of 7.2 and $12.1 \mathrm{keV}$, respectively, in combination

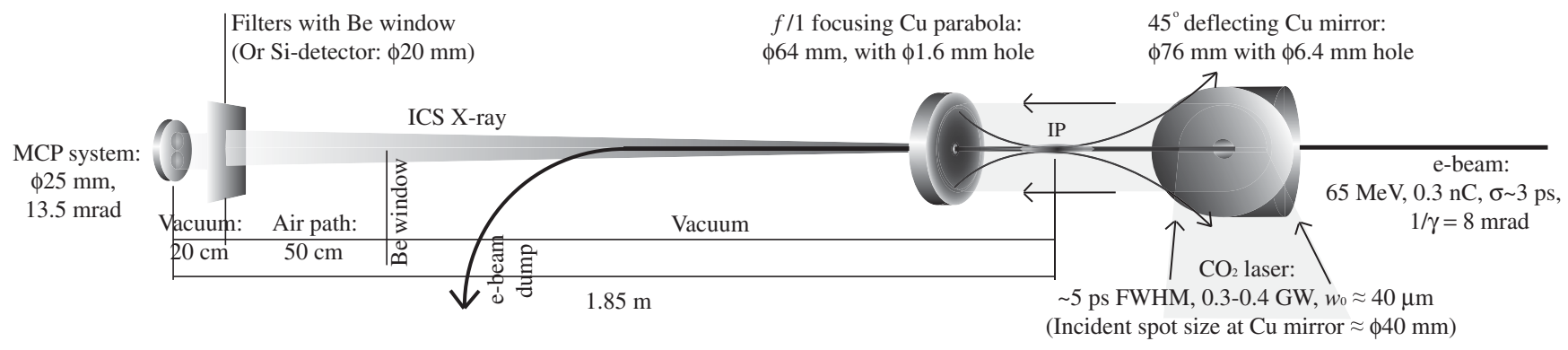

FIG. 3. Schematic layout of nonlinear ICS experiment at the BNL ATF. 


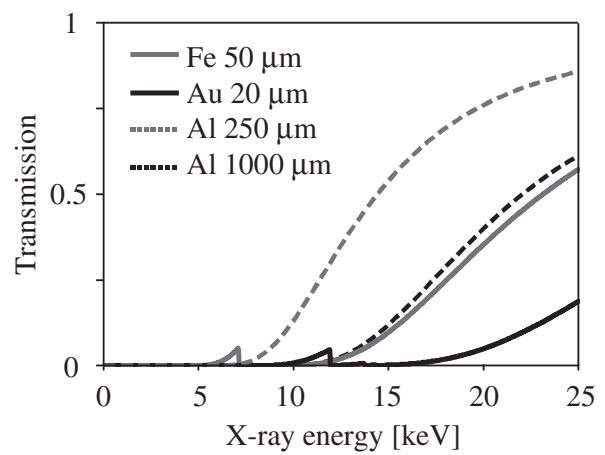

FIG. 4. Spectral transmission of filters used in the experiment, per Ref. [39]. Absorption along the $0.5 \mathrm{~m}$ air path and the $2 \times 250 \mu \mathrm{m}$ thick Be vacuum windows is included.

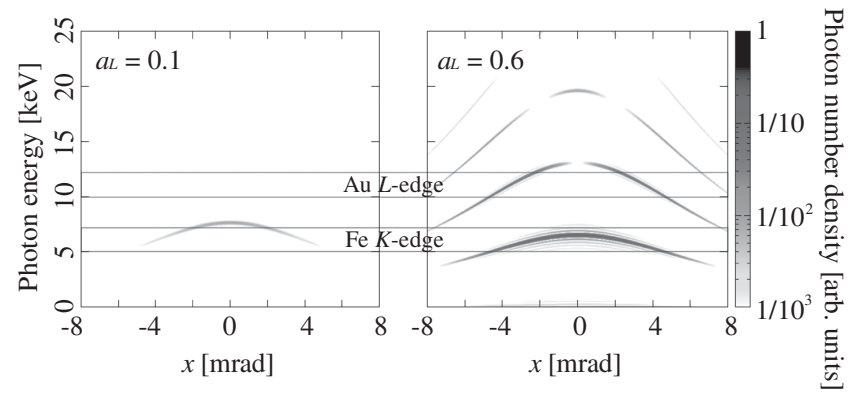

FIG. 5. Angular-frequency spectral distributions of the fundamental, 2nd and 3rd harmonics, numerically calculated for ICS emission with $a_{L}=0.1$ (left) and $a_{L}=0.6$ (right), based on a Lienard-Wiechert field potential.

with low-energy attenuating $\mathrm{Al}$ foils of $250 \mu \mathrm{m}$ and $1 \mathrm{~mm}$ thickness, respectively [39]. The $250 \mu \mathrm{m} \mathrm{Al} \mathrm{filter} \mathrm{has}$ transmittance of $\sim 0.25$ and $\sim 0.75$ for 12 and $18 \mathrm{keV}$, respectively. In the case of the $1 \mathrm{~mm} \mathrm{Al}$ filters, the transmittance is near 0 and $\sim 0.25$ for these two energies, respectively. Figure 5 illustrates how spectral filtering serves to unveil characteristic changes in the radiation pattern for ideal sinusoidal wave field. Here, the spectral and angular distributions of the lowest harmonic components, calculated for $a_{L}=0.1$ (left) and 0.6 (right), are overlaid upon the respective transmission windows.

\section{EXPERIMENTAL RESULTS}

For the $65 \mathrm{MeV}$ electron beam used in the experiments, the highest energy emitted photon in the linear regime is $7.6 \mathrm{keV}$, just above the relatively narrow $\mathrm{Fe} K$-edge transmission window [Fig. 6 (upper)]. A Fe foil placed in the X-ray beam's path to the MCP detector thus absorbs the near-axis portion of the $\mathrm{x}$-ray spectrum that exceeds the $K$-edge energy. This effect generates a characteristic annular pattern on the MCP screen, as discussed in Ref. [40]. By increasing the laser intensity to attain strong nonlinear effects at $a_{L} \approx 0.6$, the highest energy emitted (on-axis) for the fundamental component of the ICS spectrum is displaced downward to below $6.7 \mathrm{keV}$ via the relativistic
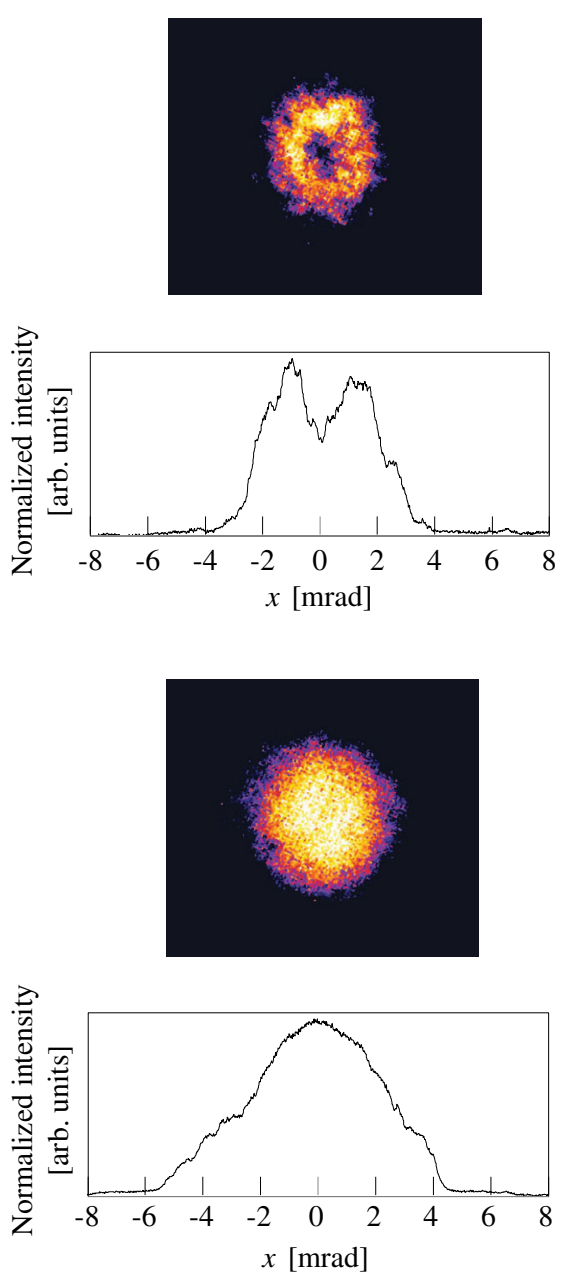

FIG. 6. Demonstration of redshifting in the ICS fundamental frequency using a Fe $K$-edge filter. Single-shot x-ray-intensity distributions were observed on the MCP screen with an upstream $50 \mu \mathrm{m}$ Fe foil (7.2 keV $K$-edge) in $10 \mathrm{mrad}$ square: (upper) $a_{L}<0.25$ case when the fundamental high-energy edge is near $7.6 \mathrm{keV}$; and (lower) $0.5<a_{L}<0.7$ case when this edge drops into the $K$-edge transmission window. The contrast is adjusted for these color-rendered MCP images. Lineout profiles correspond to the line $y=0$, giving the intensity distributions measured by the MCP.

redshift mechanism, a mass shift effect. The peak on-axis energy is then well within the Fe $K$-edge transmission window, and therefore the on-axis portion of the x-ray beam is transmitted to the screen (Fig. 6 (lower)). This observation serves as direct evidence for the relativistic redshift in the ICS spectrum. From the observed redshift, the estimated normalized vector potential given by Eq. (6) is determined to lay within the range $0.5<a_{L}<0.7$, in agreement with the expected laser parameters.

With the relativistic redshift in this nonlinear interaction established, the observation of harmonics in the ICS radiation is now described. Figure 7(a) shows the passband extraction of the 2nd harmonic, produced at photon energies in the range of $\sim 10-12 \mathrm{keV}$, by use of the 

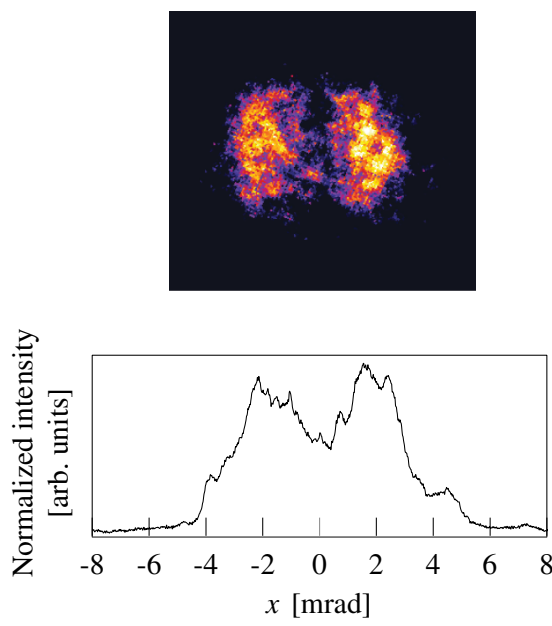

(a)
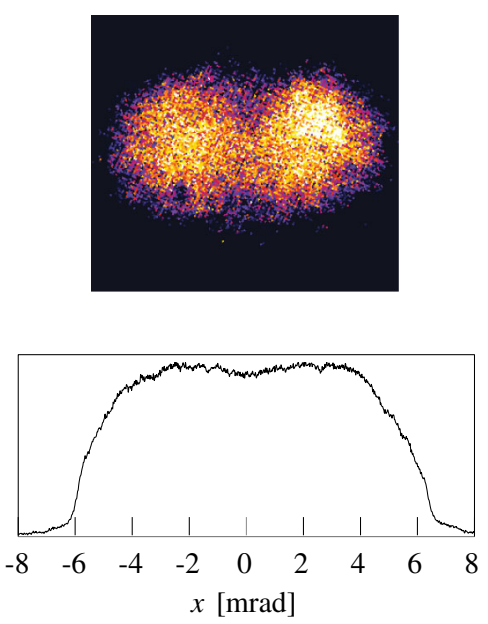

(b)
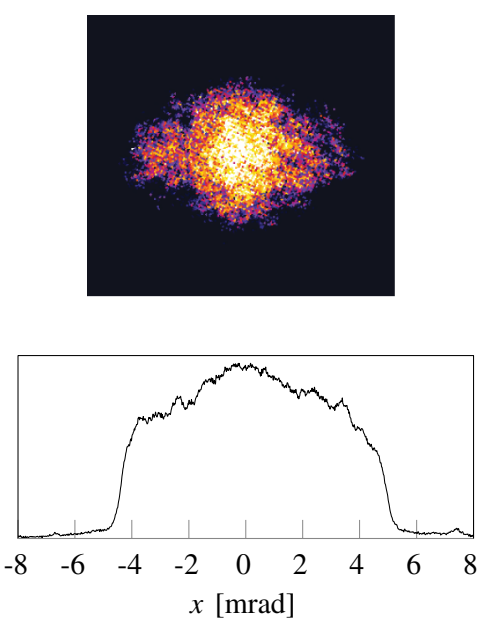

(c)

FIG. 7. Observed ICS 2nd and 3rd harmonics at MCP with the laser parameter $0.5<a_{L}<0.7$ with: (a) narrow-band transmission through $20 \mu \mathrm{m}$ Au foil giving the characteristic shape of the ICS 2nd harmonic; (b) transmission through $250 \mu \mathrm{m}$ Al foil showing the superposition of 2nd and 3rd harmonics; and (c) $1000 \mu \mathrm{m} \mathrm{Al}$ foil effectively transmitting only 3rd and higher harmonics. Contrast has adjusted for the rendered color MCP image. Lineout profiles correspond to the line $y=0$, giving the normalized intensity distributions measured.

$\mathrm{Au}$ foil filter. This measurement distinguishes two narrow crescent-shaped lobes having individual thickness of 2 mrad, separated by a half opening angle of $\sim 2 \mathrm{mrad}<1 /\left(2 \gamma_{0}\right)$, values that are estimated numerically as shown in Fig. 5 (right) at 10-12 keV. These crescent shaped lobes are, as reviewed in Sec. II, a signature of the electrons' figure-8 electron motion in the nonlinear regime. In addition to the details of angular distribution, $L$-edge filtering verifies the predicted photon energy range of ICS 2nd harmonic $[32,33]$, including the significant frequency redshift encountered in this case.

To investigate the existence of harmonics at order $>2, \mathrm{Al}$ filters of $250 \mu \mathrm{m}$ and $1 \mathrm{~mm}$ thicknesses were employed. The $250 \mu \mathrm{m} \mathrm{Al}$ filter allows one to access a relatively broader spectral range that includes the 2 nd as well as anticipated higher orders. The resulting radiation patterns observed at the MCP are shown in Figs. 7(b) and 7(c), for the $250 \mu \mathrm{m}$ and $1 \mathrm{~mm}$ filters, respectively. Figure 7(b) shows how the gap between two characteristic lobes of the 2nd harmonic component fills in noticeably, due to the angular pattern of the contribution from the 3rd harmonic. The Lienard-Wiechert model indicates that the on-axis component of the 3rd harmonic should be concentrated within an opening angle of $<1 /\left(3 \gamma_{0}\right) \operatorname{mrad}$ [Figs. 2(c) and 5 (right)]. This harmonic component is mainly induced by longitudinal motion along the strong figure- 8 motion.

Figure 7(c), with its stronger attenuation of the 2nd harmonic component due to use of the $1 \mathrm{~mm}$ thick $\mathrm{Al}$ filter, corresponds indeed to photons having contributions that arise mainly from the 3rd harmonic. The radiation pattern displayed shows the pair of side lobes surrounding a central peak having a half opening angle of $<4 \operatorname{mrad} \approx 1 /\left(2 \gamma_{0}\right)$, as seen in Figs. 2(c) and 5 (right). The observed central peak is particularly intense in the measurement, while the side lobes are off-axis redshifted and thus suffer partial attenuation. As a result, sufficient proof required to firmly establish the existence of the oscillatory longitudinal motion associated with the figure- 8 motion in strong laser field has been convincingly obtained through the geometric features of the distribution by the on-axis 3rd harmonic ICS radiation. Along with the verification of the redshifting, sufficient condition that validates the LW potential-based physics model is clarified.

By use of a Si-diode detector, the total x-ray photon number produced in this linearly polarized ICS interaction case is measured to be $\sim 10^{8}$ at the detector, and with the estimated losses, by air path and Be vacuum windows, this translates to $\sim 10^{9}$ photons at the IP. As a consequent, by employing the Si detector in conjunction with the Al high energy attenuation filters, the ratios of 1 st, 2 nd, and 3 rd harmonics of the ICS radiation was measured to be approximately $1: 1 / 25: 1 / 125$. These ratios are reasonable

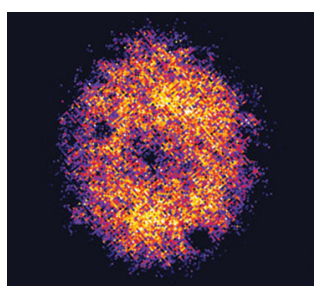

FIG. 8. Observed distribution of 2nd harmonic ICS radiation from a circular polarized laser field, with spectral band isolated using a $250 \mu \mathrm{m} \mathrm{Al}$ filter. The annular shape of the radiation distribution implies existence of a strong transverse helical motion. 
numbers based on the LW model for the Gaussian laser pulse with peak intensity of expected $0.5<a_{L}<0.7$.

Through use of a quarter-wave plate between the regenerative amplifier and the final amplifier of the $\mathrm{CO}_{2}$ laser system chain, the ICS radiation distribution obtained by scattering a circularly polarized laser in the nonlinear regime is elucidated. The resulting ICS photon distribution, which is also circularly polarized, displays an off-axis 2 nd harmonic component, which was selected by an $250 \mu \mathrm{m}$ thick Al filter. The results of this measurement are displayed in Fig. 8. In this case, the polarization symmetry should yield an angular distribution that takes on an annular shape, an effect that is indeed observed. With circular polarization, the nonlinear harmonic effects are not created by the existence of harmonics in the electron oscillatory motion, as there is no figure- 8 motion induced-there is instead strong rotational helical motion. The measured 2nd harmonic component arises instead from angular asymmetries in the helical transverse motion.

\section{CONCLUSION}

For the first time, direct observation of the nonlinear redshift in the ICS scattered photon spectrum has been accomplished in a single-shot experiment. This observed frequency redshift [21] is viewed as due to the increase in effective mass of the electron oscillating in a linearly polarized laser field. This effect, which indicates an entrance into the clearly observable nonlinear regime, has been shown through $K$-edge filter angularly resolved measurements, to be consistent with an estimated normalized vector potential $0.5<a_{L}<0.7$. With the value of $a_{L}$, approaching to unity, this nonlinear effect is accompanied by the extension of higher harmonic emission up to 3rd order. These 2nd and 3rd order harmonics were verified in the expected spectral bands. The spectral-angular distributions observed directly indicates the existence of the figure8 motion induced to the electron's oscillatory longitudinal momentum in a relativistically intense, linearly polarized laser field.

The exploration of two notable nonlinear effects in ICS has permitted the qualitative validation of the electrodynamics model of the radiation production. This observation opens the door to further experimental investigation which exploits the control of the relativistic radiation kinetics in the $x-, \gamma$-ray regimes using nonlinearities. One may, for example, wish to either enhance or suppress harmonics in the emitted spectrum, or realize harmonics mixing with arbitrary polarizations [34]. In regards to the related interest of time encoded spectrum modulation that occurs during ps $\mathrm{CO}_{2}$ laser pulse, applications of nonlinear ICS pulses to pump probe experiments as initial proof of principle experiments are under consideration at BNL ATF. This development takes place in the context of the UCLA project to create an all-optical, quasimonochromatic ICS/IFEL $\gamma$-ray source. These following steps would serve to help optimize tunable, ps-to-fs ICS photon production as utilized in a wide variety of emerging applications, at higher photon energy, and clarify experimental understanding of strong field laser-electron interaction physics.

The results obtained here are general, and are relevant to all Compton sources that operate in the Thomson limit, where the recoil of the electron in its nominal rest frame is negligible. The results would be applied to the applications discussed in the Introduction, up to $\mathrm{MeV}$ or higher photon energy. Further studies that deepen the understanding of the basic electrodynamics will arise from future experiments with a range of collision scenarios, such as variations in laser polarization and incidence angle, and use of higher energy electron beams.

\section{ACKNOWLEDGMENTS}

This work was supported by U.S. DHS DNDO under Contract No. 2014-DN-077-ARI084-01, DTRA under Contract No. HDTRA1-10-1-0073, the ONR under Contract No. ONR N00014-06-1-0925, DARPA under Contract No. N66001-11-1-4197 and U.S. DOE under Contracts No. DE-FG02-07ER46272, No. DEFG03-92ER40693, No. DE-AC02-98CH10886. Authors would like to thank all collaborators in BNL ATF and UCLA PBPL. We would acknowledge the invaluable special advice and contribution from Dr. L. Shao, Dr. X. Ding, Dr. D. Cline, Dr. S. Tochitsky (UCLA), Dr. P. Siddons (BNL), Dr. Y. Kamiya (Tokyo University) and Dr. T. Kumita (Tokyo Metropolitan University).

[1] G. R. Blumenthal and R. J. Gould, Rev. Mod. Phys. 42, 237 (1970).

[2] P. Sprangle, A. Ting, E. Esarey, and A. Fisher, J. Appl. Phys. 72, 5032 (1992).

[3] W. P. Leemans, R. W. Schoenlein, P. Volfbeyn, A. H. Chin, T. E. Glover, P. Balling, M. Zolotorev, K. J. Kim, S. Chattopadhyay, and C. V. Shank, Phys. Rev. Lett. 77, 4182 (1996).

[4] I. V. Pogorelsky et al., Phys. Rev. ST Accel. Beams 3, 090702 (2000).

[5] M. Uesaka et al., Nucl. Instrum. Methods Phys. Res., Sect. A 455, 90 (2000).

[6] S. Kashiwagi et al., J. Appl. Phys. 98, 123302 (2005).

[7] A. M. Tremaine et al., in Proceedings of the 21st Particle Accelerator Conference, Knoxville, TN, 2005 (IEEE, Piscataway, NJ, 2005), p. 1464.

[8] G. B. Rybick and A. P. Lightman, Radiative Processes in Astrophysics (John Wiley \& Sons, Inc., New York, 1991).

[9] R. Kuroda et al., Nucl. Instrum. Methods Phys. Res., Sect. A 637, S183 (2011).

[10] F. H. O'Shea, O. Williams, G. Andonian, S. Barber, Y. Sakai, J. B. Rosenzweig, I. Pogorelsky, M. Fedurin, K. Kusche, and V. Yakimenko, Phys. Rev. ST Accel. Beams 15, 020702 (2012). 
[11] A. Murokh et al., in Proceedings of the 3rd International Particle Accelerator Conference, New Orleans, LA, 2012 (IEEE, Piscataway, NJ, 2012), THEPPB008.

[12] R. Eichhorn et al., in Proceedings of International Particle Accelerator Conference, Germany (2014), WEPRI061.

[13] T. Omori et al., Phys. Rev. Lett. 96, 114801 (2006).

[14] L. J. Nevay, S. T. Boogert, P. Karataev, K. Kruchinin, L. Corner, D. F. Howell, R. Walczak, A. Aryshev, J. Urakawa, and N. Terunuma, Phys. Rev. ST Accel. Beams 17, 072802 (2014).

[15] V. Telnov, Nucl. Instrum. Methods Phys. Res., Sect. A 455, 63 (2000).

[16] E. C. Schreiber et al., Phys. Rev. C 61, 061604 (2000).

[17] D. J. Gibson et al., Phys. Rev. ST Accel. Beams 13, 070703 (2010).

[18] S. Chen et al., Phys. Rev. Lett. 110, 155003 (2013).

[19] J. Duris et al., Nat. Commun. 5, 4928 (2014).

[20] L. S. Brown and T. W. B. Kibble, Phys. Rev. 133, A705 (1964).

[21] T. W. B. Kibble, Phys. Rev. 138, B740 (1965).

[22] E. S. Sarachik and G. T. Schappert, Phys. Rev. D 1, 2738 (1970).

[23] K. T. MacDonald, Proposal for experimental studies of nonlinear electrodynamics, Princeton University Report No. DOE/ER/3072-38, 1986.

[24] E. Esarey, S. K. Ride, and P. Sprangle, Phys. Rev. E 48, 3003 (1993).

[25] G. A. Krafft, A. Doyuran, and J. B. Rosenzweig, Phys. Rev. E 72, 056502 (2005).
[26] T. Heinzl, D. Seipt, and B. Kämpfer, Phys. Rev. A 81, 022125 (2010).

[27] F. Mackenroth and A. Di Piazza, Phys. Rev. A 83, 032106 (2011).

[28] T. J. Englert and E. A. Rinehart, Phys. Rev. A 28, 1539 (1983).

[29] C. Bula et al., Phys. Rev. Lett. 76, 3116 (1996).

[30] D. D. Meyerhofer, J. P. Knauer, S. J. McNaught, and C. I. Moore, J. Opt. Soc. Am. B 13, 113 (1996).

[31] S. Y. Chen, A. Maksimchuk1, and D. Umstadter, Nature (London) 396, 653 (1998).

[32] M. Babzien et al., Phys. Rev. Lett. 96, 054802 (2006).

[33] T. Kumita et al., Laser Phys. 16, 267 (2006).

[34] Y. Sakai, O. Williams, G. Andonian, A. Fukasawa, E. Hemsing, A. Marinelli, S. Barber, F. H. O'Shea, and J. B. Rosenzweig, Phys. Rev. ST Accel. Beams 14, 120702 (2011).

[35] L. D. Landau and E. M. Lifshitz, The Classical Theory of Fields (Pergamon Press Ltd., New York, 1951).

[36] H. Motz, J. Appl. Phys. 22, 527 (1951).

[37] J. D. Jackson, Classical Electrodynamics (Wiley, New York, 1962).

[38] M. N. Polyanskiy, I. V. Pogorelsky, and V. Yakimenko, Opt. Express 19, 7717 (2011).

[39] B. L. Henke, E. M. Gullikson, and J. C. Davis, At. Data Nucl. Data Tables 54, 181 (1993).

[40] O. Williams, G. Andonian, M. Babzien, E. Hemsing, K. Kusche, J. Park, I. Pogorelsky, G. Priebe, J. Rosenzweig, and V. Yakimenko, Nucl. Instrum. Methods Phys. Res., Sect. A 608, S18 (2009). 